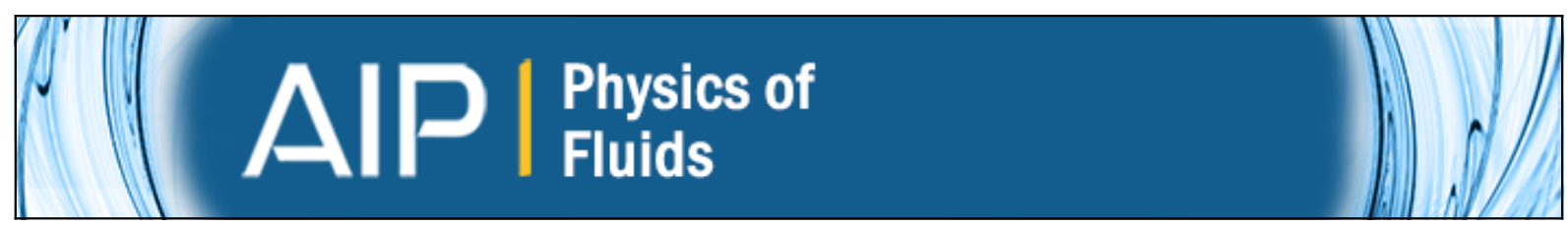

\title{
Close pairs of relative equilibria for identical point vortices
}

Tobias Dirksen and Hassan Aref

Citation: Physics of Fluids (1994-present) 23, 051706 (2011); doi: 10.1063/1.3590740

View online: http://dx.doi.org/10.1063/1.3590740

View Table of Contents: http://scitation.aip.org/content/aip/journal/pof2/23/5?ver=pdfcov

Published by the AIP Publishing

Copyright by the American Institute of Physics. Close pairs of relative equilibria for identical point vortices. Dirksen, Tobias and Aref, Hassan, Physics of Fluids (1994present), 23, 051706 (2011), DOI $:$ http: //

dx.doi.org/10.1063/1.3590740

\section{AlP Re-register for Table of Content Alerts}




\title{
Close pairs of relative equilibria for identical point vortices
}

\author{
Tobias Dirksen ${ }^{1, a)}$ and Hassan Aref $^{2,3, b)}$ \\ ${ }^{1}$ Department of Physics, Technical University of Denmark, Lyngby, Denmark \\ ${ }^{2}$ Center for Fluid Dynamics, Technical University of Denmark, Lyngby, Denmark \\ ${ }^{3}$ Engineering Science and Mechanics, Virginia Tech, Blacksburg, Virginia 24061, USA
}

(Received 16 March 2011; accepted 20 April 2011; published online 23 May 2011)

\begin{abstract}
Numerical solution of the classical problem of relative equilibria for identical point vortices on the unbounded plane reveals configurations that are very close to the analytically known, centered, symmetrically arranged, nested equilateral triangles. New numerical solutions of this kind are found for $3 n+1$ vortices, where $n=2,3, \ldots, 30$. A sufficient, although apparently not necessary, condition for this phenomenon of close solutions is that the "core" of the configuration is marginally stable, as occurs for a central vortex surrounded by an equilateral triangle. The open, regular heptagon also has this property, and new relative equilibria close to the nested, symmetrically arranged, regular heptagons have been found. The centered regular nonagon is also marginally stable. Again, a new family of close relative equilibria has been found. The closest relative equilibrium pairs occur, however, for symmetrically nested equilateral triangles. () 2011 American Institute of Physics. [doi:10.1063/1.3590740]
\end{abstract}

The problem of relative equilibria of identical point vortices goes back to work by Mayer, W. Thomson (the later Lord Kelvin), and J. J. Thomson in the 1870s. ${ }^{1}$ This problem, and closely related problems, arise in a variety of contexts. ${ }^{2}$

The equations to be solved are as follows: Let the vortex positions be given as $N$ points in the complex plane, $z_{1}, \ldots, z_{N}$. To find all solutions of the system ${ }^{1}$

$$
\bar{z}_{\alpha}=\sum_{\beta=1}^{N}, \frac{1}{z_{\alpha}-z_{\beta}}, \quad \alpha=1, \ldots, N .
$$

The overbar on the left signifies complex conjugation, the prime on the summation sign that $\beta \neq \alpha$.

Several numerical explorations have been undertaken, the most comprehensive being the Los Alamos Catalog compiled in the late 1970s, henceforth referred to simply as the Catalog. ${ }^{3}$ It was unfortunately never published in the archival literature although many of the results may be found in a companion paper. ${ }^{4}$ The Catalog was inspired by the first visualizations ${ }^{5}$ of relative equilibria in He II, and so emphasized linearly stable configurations. When one expands the search to all relative equilibria, a large number of unstable configurations are found. Thus, for $N=4$, there is just one entry in the Catalog, a square of vortices. Two additional configurations are known: the marginally stable, centered, equilateral triangle, and an unstable, collinear configuration with the vortex positions at the roots of the Hermite polynomial $H_{4}$. A configuration close to the centered equilateral triangle, known for the 4-body problem of celestial mechanics, ${ }^{6}$ appears not to arise for point vortices. For $N=5$, both the regular pentagon and the centered square are linearly stable (and in the Catalog), but there are three additional unstable relative equilibria. For $N=6$, eight relative equi-

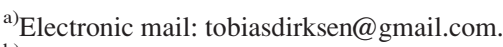

${ }^{\mathrm{b})}$ Electronic mail: haref@vt.edu.
}

libria are known of which six are unstable. The regular hexagon and the centered, regular pentagon are the only two stable configurations - and the only two reported in the Catalog.

For $N=7$, we know 11 relative equilibria, although just one of these, the centered, regular hexagon is linearly stable. Here, we report an additional configuration, found numerically, the smallest of a family of relative equilibria that are extremely close to the analytically known centered, symmetric, nested equilateral triangle configurations. All 12 relative equilibria are shown in Fig. 1 arranged by decreasing values of

$$
\theta=\frac{2}{N(N-1)} \sum_{\alpha, \beta=1}^{N}{ }^{\prime} \log \left|z_{\alpha}-z_{\beta}\right| .
$$

(The kinetic energy of interaction of the vortices is proportional to $-\theta$.) Some of these configurations are known analytically, such as the centered hexagon, Fig. 1(a), and the marginally stable, regular heptagon, Fig. 1(b), both included in the Catalog. Of the remaining configurations, the centered, triple digon, ${ }^{7}$ Fig. $1(\mathrm{~g})$, the centered, symmetric doublering, ${ }^{8}$ Fig. 1(j), and the collinear configuration with vortices at the zeros of $H_{7}$, Fig. 1(1), are known analytically.

We have computed these states using MATLAB to solve Eq. (1) in double precision (of which ten digits are given in the results reported). Once a solution is obtained, we check that it verifies the convergence criterion

$$
\frac{1}{N} \sum_{\alpha=1}^{N}\left|\bar{z}_{\alpha}-\sum_{\beta=1}^{N}{ }^{\prime} \frac{1}{z_{\alpha}-z_{\beta}}\right|<\varepsilon,
$$

where $\varepsilon$ is a convergence parameter that we typically set to $10^{-13}$. For $N=7$, the computations revealed a new configuration, Fig. 1(i). To the naked eye, this new configuration is identical to the centered, symmetric double-ring, Fig. 1(j), with vortex positions 


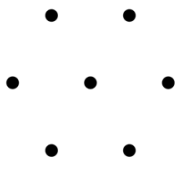

(a)

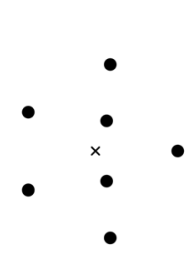

(d)

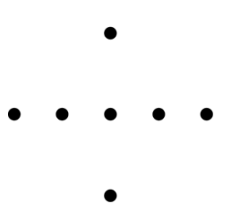

(g)

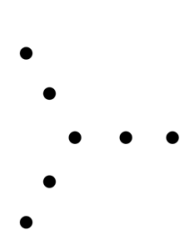

(j)

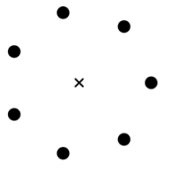

(b)

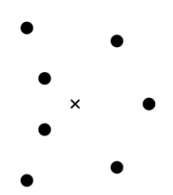

(e)

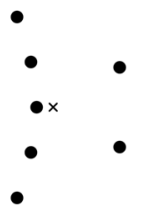

(h)

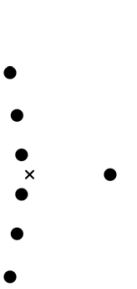

(k)

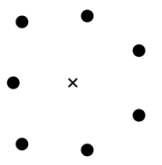

(c)

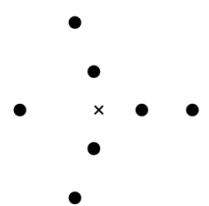

(f)

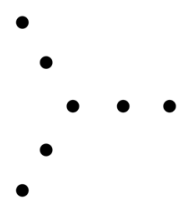

(i)
FIG. 1. Numerically determined relative equilibria of seven vortices. Centers of rotation indicated by small crosses (when not coincident with a vortex).

$$
\begin{array}{ll}
1.2251565538, & -0.6125782769 \pm 1.0610166992 \mathrm{i}, \\
2.3449928398, & -1.1724964199 \pm 2.0308233710 \mathrm{i},
\end{array}
$$

and 0 . These coordinates are just $R_{1}, R_{1} \mathrm{e}^{ \pm \mathrm{i} 2 \pi / 3}$, and $R_{2}$, $R_{2} \mathrm{e}^{ \pm \mathrm{i} 2 \pi / 3}$ (and 0 ), where $R_{1}$ and $R_{2}$ satisfy ${ }^{7,8}$

$$
R_{1}^{2}+R_{2}^{2}=7, \quad R_{1}^{5}+R_{2}^{5}=5\left(R_{1}^{3}+R_{2}^{3}\right) .
$$

The value of $\theta$, Eq. (2), for this configuration is $1.6035139510 \ldots$ in agreement with analysis. ${ }^{9}$ For the new solution, Fig. 1(i), the computed vortex positions are

$$
\begin{array}{ll}
1.2350850139 & -0.6172513235 \pm 1.0524323669 \mathrm{i}, \\
2.3511504807 & -1.1891361698 \pm 2.0175029435 \mathrm{i}, \\
0.0265394920 . &
\end{array}
$$

The value of $\theta$, Eq. (2), is $1.6035146448 \ldots$ In a numerical exploration where the existence of relative equilibria different from those seen previously is assessed visually from a plot of vortex positions, or even from a calculation of an energy measure equivalent to $\theta$ in Eq. (2) but only to accuracy $10^{-6}$, one could easily mistake the new configuration for the known, centered double-ring.
Initially, we were suspicious that the new configuration was a numerical artifact even though the stringent check (3) had been applied. Hence, we also checked the calculations to accuracy $10^{-300}$ using Maple and found both solutions to be present. These checks confirm the existence of two very close relative equilibria.

Another argument in favor of the existence of the new configuration is that a similar pair occurs close to the centered, symmetric triple-ring configuration 1-3-3-3, which is again known analytically. ${ }^{7}$ The configuration consists of a central vortex and three concentric, symmetrically placed, equilateral triangles of vortices. The vortex coordinates in this relative equilibrium are

$$
\begin{array}{ll}
1.1257068936, & -0.5628534468 \pm 0.9748907671 \mathrm{i}, \\
2.0668001012, & -1.0334000506 \pm 1.7899013922 \mathrm{i}, \\
3.0758935826, & -1.5379467913 \pm 2.6638019818 \mathrm{i},
\end{array}
$$

and 0. These coordinates are $R_{p} \mathrm{e}^{\mathrm{i} 2 \pi \alpha / 3}, \quad p=1,2,3$, and $\alpha=1,2,3$, where $R_{1}, R_{2}$, and $R_{3}$ satisfy the equations ${ }^{7}$

$$
\begin{aligned}
& R_{1}^{2}+R_{2}^{2}+R_{3}^{2}=15, \\
& R_{1}^{5}+R_{2}^{5}+R_{3}^{5}=8\left(R_{1}^{3}+R_{2}^{3}+R_{3}^{3}\right), \\
& \frac{R_{1}^{2}-2}{R_{1}^{3}}+\frac{R_{2}^{2}-2}{R_{2}^{3}}+\frac{R_{3}^{2}-2}{R_{3}^{3}}=0 .
\end{aligned}
$$

The value of $\theta$, Eq. (2), is $1.8554011511 \ldots$ The coordinates in the new relative equilibrium are

$$
\begin{array}{ll}
1.1430054923, & -0.5635151208 \pm 0.9642351902 \mathrm{i}, \\
2.0782582001, & -1.0475637987 \pm 1.7748721082 \mathrm{i}, \\
3.0842676267, & -1.5635635748 \pm 2.6439155247 \mathrm{i}, \\
0.0437536693 . &
\end{array}
$$

For this configuration, we find $\theta=1.8554026277 \ldots$

We found other instances of close relative equilibria for ten vortices, although none as close as the pair just given. The two similar configuration pairs in Figs. 2(a)-2(d) can be distinguished by careful visual inspection. Relative (a)

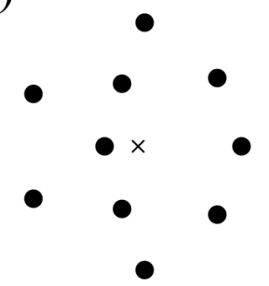

(c)

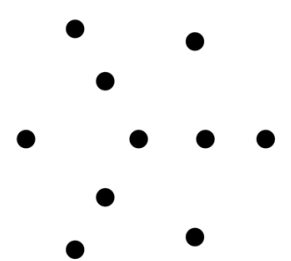

(b)

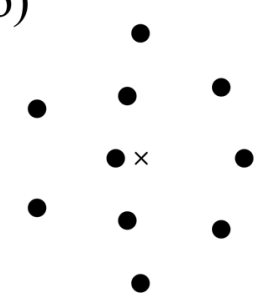

(d)

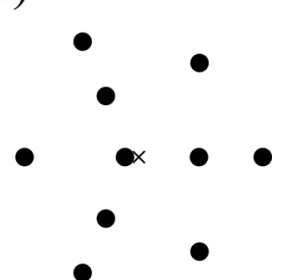

FIG. 2. Two examples, (a), (b) and (c), (d), of pairs of close relative equilibria for ten vortices. 
differences in individual coordinates are larger than in the previous examples. The values of $\theta$ for the two configurations in each pair in Fig. 2 agree to accuracy $10^{-3}$. The pair in Figs. 2(c) and 2(d) is the most interesting for the present discussion since Fig. 2(c) is a centered, staggered configuration of nested equilateral triangles in the nomenclature of Ref. 7 .

There appear to be centered, symmetric configurations of arbitrarily many nested, similar, regular polygons. For $n$ nested regular $s$-gons, we find the following system of equations for the radii $R_{1}, \ldots, R_{n}$ :

$$
\frac{2 R_{p}^{2}-s-1}{R_{p}^{s}}=2 s \sum_{q=1}^{n}{ }^{\prime} \frac{1}{R_{p}^{s}-R_{q}^{s}}, \quad p=1, \ldots, n .
$$

The prime on the summation sign means $q \neq p$. The vortex positions in such a relative equilibrium are $R_{p} \mathrm{e}^{\mathrm{i} 2 \pi \alpha / s}$, $p=1, \ldots, n, \alpha=1, \ldots, s$, and 0 . Equations (4) and (5) are reduced versions of Eqs. (6) for $s=3$ and $n=2$ and 3 , respectively. For $s=2$, Eqs. (6) are satisfied by the zeros of the Hermite polynomial of degree $2 n+1$. We shall not pause to derive Eqs. (6) here. A derivation can be based on the generating polynomial approach explained in Ref. 11, in particular, Eq. (2.6).

For $s=3$, we have found solutions to Eq. (6) for $n$ up to 30 numerically. We assume there are solutions for all natural numbers $n$. These describe configurations of $3 n+1$ vortices arranged on $n$ symmetrically nested, equilateral triangles with a vortex at the center. Based on the results for $n=2,3$, we expect that close to each of these symmetric relative equilibria there is a second relative equilibrium with lower symmetry. We have checked this conjecture for $n=4, \ldots, 30$. In each case, we find a new, close relative equilibrium that is indistinguishable from the symmetric configuration to the naked eye. The left panel of Fig. 3 provides an example for $n=10$. The vortices in the analytically known configuration are shown as black dots centered on the vortex positions. The new, close configuration is shown by superimposed white dots centered on the new vortex positions. All dots of the new configuration are seen to fall inside the black dots representing the analytically known configuration.

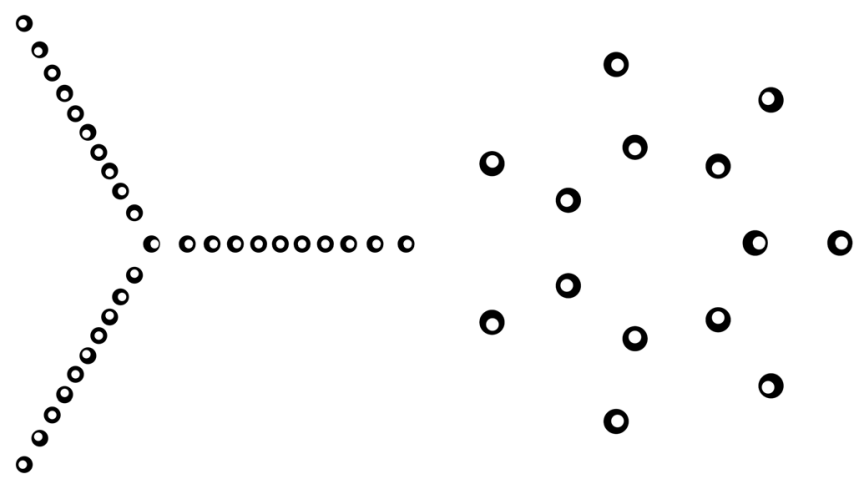

FIG. 3. Close relative equilibria for 31 (left) and 14 (right) vortices. The analytical, symmetrically-nested, regular-polygon solution is shown as black dots, the close, new, numerically determined solution is shown as superimposed white dots. These results have been checked to accuracy $10^{-300}$ using Maple.
With the new seven-vortex configuration added to the list, the number of known relative equilibria of seven identical vortices stands at 12 . There are 19 known eight-vortex relative equilibria. The total could also be stated as 20 since for $N=8$, we encounter the smallest asymmetric relative equilibrium. ${ }^{10} \mathrm{~A}$ reflection of this configuration in a line through its centroid is again a relative equilibrium and not one that can be obtained by rotation from the original configuration.

For nine vortices, we found a total of 35 relative equilibria. Four of these are asymmetric so one could count each of them as two different configurations for a total of 39. For ten vortices, we found 59 relative equilibria of which 13 are asymmetric. We have not yet done a systematic count for larger values of $N$. For $N=13$, where we were interested in whether there was another relative equilibrium close to the symmetric 1-3-3-3-3 configuration, we found at least 275 relative equilibria. The total number of relative equilibria appears to grow rapidly with $N$, and the number of asymmetric configurations among them also grows.

Let $z_{\alpha}^{(0)}$ and $z_{\alpha}$ be two close relative equilibria. To linear order in $\delta z_{\alpha}=z_{\alpha}-z_{\alpha}^{(0)}$, we have

$$
\bar{\delta}_{\alpha}=\sum_{\beta=1}^{N} A_{\alpha \beta} \delta z_{\beta},
$$

where the matrix $A_{\alpha \beta}$ is the matrix that arises in the linear stability theory for the configuration $z_{\alpha}^{(0)}$

$$
A_{\alpha \beta}=\sum_{\gamma=1}^{N}, \frac{\delta_{\alpha \beta}}{\left(z_{\alpha}^{(0)}-z_{\gamma}^{(0)}\right)^{2}}-\frac{1-\delta_{\alpha \beta}}{\left(z_{\alpha}^{(0)}-z_{\beta}^{(0)}\right)^{2}} .
$$

We write Eq. (7a) in an easily understood vector-matrix notation

$$
\overline{\delta z}=\boldsymbol{A} \delta z, \quad \delta z=\overline{\boldsymbol{A}} \boldsymbol{A} \delta z
$$

Since $\boldsymbol{A}$ is symmetric, $\overline{\boldsymbol{A}} \boldsymbol{A}$ is Hermitian. Furthermore, because of the form of this matrix, all its eigenvalues are non-negative. The existence of a close relative equilibrium suggests that the matrix $\overline{\boldsymbol{A}} \boldsymbol{A}$ must have an eigenvector with eigenvalue 1 . Now, $z^{(0)}$ is always a "trivial" eigenvector of $\overline{\boldsymbol{A}} \boldsymbol{A}$ with eigenvalue 1 (cf. Ref. 12 where the analogous result for $N=3$ and arbitrary vortex strengths is given). Thus, we expect that a close pair of relative equilibria will manifest itself by the matrix $\overline{\boldsymbol{A}} \boldsymbol{A}$ having a close degeneracy of the eigenvalue 1 .

We have computed the eigenvalues of $\overline{\boldsymbol{A}} \boldsymbol{A}$ for each of the configurations found. The eigenvalue spectra for close configurations are, of course, also close. For example, in the case of Figs. 1(i) and 1(j), the eigenvalue spectrum of the analytically known configuration is $0.0000,1.0000,1.0456$ (multiplicity 2), 5.5157 (multiplicity 2), and 6.1539. The eigenvalues of the new configuration are $0.0000,0.9555$, $1.0000,1.1396,5.3727,5.6504$, and 6.1674. (The eigenvalue 0 is also always present, cf. Ref. 12.) The pattern of the less symmetrical configuration having a stable mode with eigenvalue less than but somewhat close to 1 recurs for the other close pairs. 
A related observation is that the eigenvalue spectrum of the centered, equilateral triangle is 0,1 (with multiplicity 3 ). This suggests a very "flat" energy surface around this state. As further equilateral triangles are added, this degeneracy in the stability matrix for the "core" of the configuration may be the main feature that allows a close relative equilibrium to exist.

Among the open and centered regular polygons, the open heptagon and centered nonagon are both marginally stable in linear theory, i.e., the eigenvalue 1 of $\overline{\boldsymbol{A}} \boldsymbol{A}$ is the largest and is degenerate. Hence, one suspects that close to the heptagon double-ring, there would be another close relative equilibrium of lower symmetry. We have checked this conjecture and, indeed, such a configuration is found numerically. It is shown in the right panel in Fig. 3, where the analytically known double ring ${ }^{7,8}$ is plotted using black dots, the new numerically determined, close, 14 -vortex relative equilibrium using superimposed white dots. The two configurations are obviously very close, although not quite as close as in the case of the nested equilateral triangles. Further, close relative equilibrium pairs exist as more heptagonal rings are added. The ring radii in the analytical solutions satisfy equations similar to Eq. (6) but with $-s+1$ in the numerator instead of $-s-1$ (and for heptagons $s=7$ ). Similarly, for the centered, regular nonagons we have found relative equilibria that are close to the centered double- and triple-rings but, again, not as close as in the case of the equilateral triangles. The centered equilateral triangle is unique in that all infinitesimal perturbations with fixed centroid have eigenvalue 1 . The open regular heptagon and centered regular nonagon also have linearly stable modes.

We do not have a full understanding of why the phenomenon of close pairs of solutions to Eq. (1) arises. The closest solutions appear in the vicinity of the centered, symmetric, nested equilateral triangle configurations. Close solutions also occur in other cases when the "core" of the configuration is marginally stable and the eigenvalue 1 has multiplicity larger than 1 . However, quite close solutions, such as Figs. 2(a) and 2(b), arise in cases where this mechanism of a marginally stable "core" does not seem to be applicable. The phenomenon of close relative equilibria was a surprise to us. We have since learned of similar situations in other problems of this kind. ${ }^{6,13,14}$ For relative equilibria with vortices of both positive and negative circulations examples of continua of solutions are known, e.g., for the stationary relative equilibria ${ }^{15}$ and for a certain five-vortex example. ${ }^{16}$ However, the expectation is that when all vortices are of one sign, in particular, for identical vortices, "finiteness" of solutions prevails. Implicit in this expectation was that the different relative equilibria would be easily distinguishable.

We thank B. Eckhardt, R. Hansen, K. O'Neil, and D. Vainchtein for discussions and comments. This work was supported in part by a Niels Bohr Visiting Professorship at the Technical University of Denmark sponsored by the Danish National Research Foundation.

${ }^{1}$ H. Aref, P. K. Newton, M. A. Stremler, T. Tokieda, and D. L. Vainchtein, "Vortex crystals," Adv. Appl. Mech. 39, 1 (2003).

${ }^{2}$ P. K. Newton and G. Chamoun, "Vortex lattice theory: A particle interaction perspective," SIAM Rev. 51, 501 (2009).

${ }^{3}$ L. J. Campbell and R. M. Ziff, "A catalog of two-dimensional vortex patterns," Rev. Informal Report LA-7384-MS, Los Alamos National Laboratory, 1978, pp.1-40.

${ }^{4}$ L. J. Campbell and R. M. Ziff, "Vortex patterns and energies in a rotating superfluid," Phys. Rev. B 20, 1886 (1979).

${ }^{5}$ E. J. Yarmchuk, M. J. V. Gordon, and R. E. Packard, "Observation of stationary vortex arrays in rotating superfluid Helium," Phys. Rev. Lett. 43, 214 (1979).

${ }^{6} \mathrm{~K}$. Glass, "Equilibrium configurations for a system of $N$ particles in the plane," Phys. Lett. A 235, 591 (1997).

${ }^{7}$ H. Aref and M. van Buren, "Vortex triple rings," Phys. Fluids 17, 057104, (2005).

${ }^{8}$ T. H. Havelock, "Stability of motion of rectilinear vortices in ring formation," Philos. Mag. 11, 617 (1931).

${ }^{9} \mathrm{H}$. Aref, "A note on the energy of relative equilibria of point vortices," Phys. Fluids 19, 103603 (2007).

${ }^{10} \mathrm{H}$. Aref and D. L. Vainchtein, "Asymmetric equilibrium patterns of point vortices," Nature 392, 769 (1998).

${ }^{11} \mathrm{H}$. Aref, "Relative equilibria of point vortices and the fundamental theorem of algebra," Proc. R. Soc. London, Ser. A (in press).

${ }^{12} \mathrm{H}$. Aref, "Stability of relative equilibria of three vortices," Phys. Fluids 21, 094101 (2009).

${ }^{13}$ K. Sacha and B. Eckhardt, "Pathways to non-sequential multiple ionization in strong laser fields," J. Phys. B 36, 3293 (2003).

${ }^{14} \mathrm{~K}$. A. O'Neil, "Central configurations of identical masses lying along curves," Phys. Rev. E 79, 066601 (2009).

${ }^{15}$ A. B. Bartman, "A new interpretation of the Adler-Moser KdV polynomials: Interaction of vortices," in Nonlinear and Turbulent Processes in Physics, edited by R. Z. Sagdeev (Harwood Academic, Newark, NJ, 1984), Vol. 3, pp. 1175-1181.

${ }^{16} \mathrm{G}$. E. Roberts, "A continuum of relative equilibria in the five-body problem," Physica D 127, 141 (1999). 